\title{
Medical Image of the Week: Lactate Threshold
}

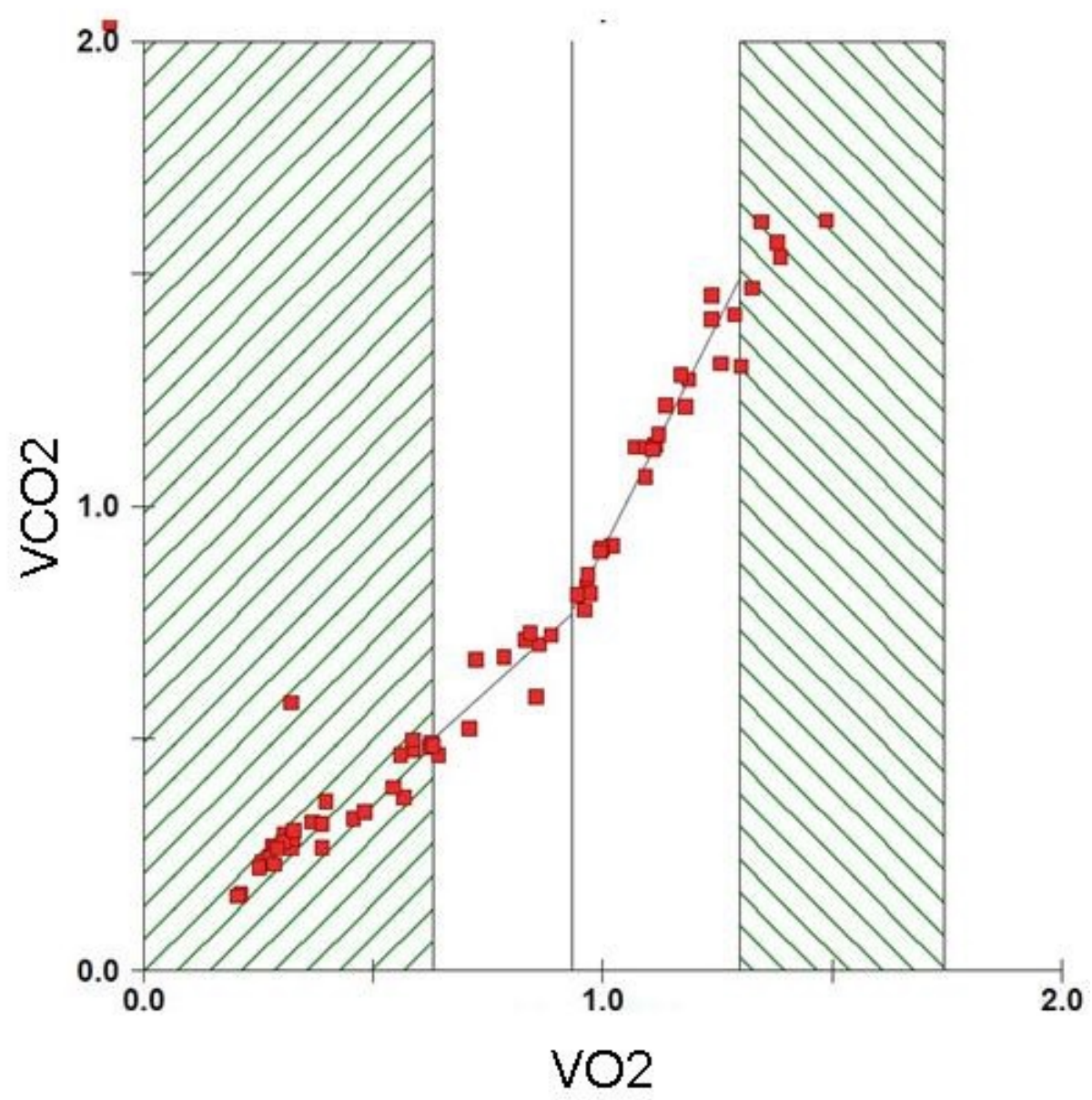

Figure 1. V slope method for lactate threshold is where the CO2 production (VCO) increases compared to oxygen consumption (VO2). This is indicated by the vertical line.

The lactate or anaerobic threshold in exercise physiology is the point at which lactic acid starts to accumulate as the anaerobic component of metabolism increases and has been used to assess cardiopulmonary fitness. The $V$-slope method is a non-invasive way of estimating the lactate or anaerobic threshold (1). As lactate accumulates the $\mathrm{H}+$ is mainly buffered by $\mathrm{HCO}$ - and produces $\mathrm{CO} 2$. A point can be seen in Figure 1 where $\mathrm{CO} 2$ production (VCO2) abruptly increases relative to O2 consumption (VO2). This represents the point at which the lactate threshold occurs.

Mohammad R. Dalabih, MBBS and Naser J. Mahmoud, MBBS

Division of Pulmonary, Allergy and Critical Care

The University of Arizona

Tucson, AZ USA

\section{Reference}

1. Beaver WL, Wasserman K, Whipp BJ. A new method for detecting anaerobic threshold by gas exchange. J Appl Physiol. 1986;60(6):2020-7. [PubMed] 